\title{
Self-consistent Study on Color Transport in the Quark Gluon Plasma at Finite Chemical Potential
}

\author{
Hou Defu ${ }^{\dagger}$, Stefan Ochs ${ }^{* \dagger}$, Li Jiarong ${ }^{\dagger}$ \\ September 24, 2018 \\ †'Institute of Particle Physics, Hua-Zhong Normal University \\ Wuhan 430070, China \\ *Institut für theoretische Physik, 93040 Uni Regensburg, Germany
}

\begin{abstract}
We calculate the relaxation time self-consistently to study the damping of collective color modes and the color conductivity in a QGP by deriving self-consistent equations for the damping rates of gluons and quarks to leading order QCD by TFD including a chemical potential for quarks. We show that the damping rates are not sensitive to the chemical potential whereas color conductivity is enhanced considerably.
\end{abstract}

\section{Introduction}

Heavy ion experiments at RHIC and SPS have now reached a stage where we can expect to probe QCD matter beyond its hadronic state in the quark gluon plasma (QGP) phase. For this phase of essentially free colored particles we expect a behaviour similar to the one in a QED plasma, that is Debye screening of color electric charges and their interactions, as well as collective color modes [1], [2].

Collective behaviour might be observable in ultrarelativistic heavy ion collisions as long as the lifetime of the QGP exceeds the time scale for dissipative processes. Therefore it is important to calculate the damping rate for collective phenomena.

So far, mostly momentum relaxation rates were considered [3], 泊], which allow then estimates for viscosity, thermal conductivity and energy transport in the plasma. Some

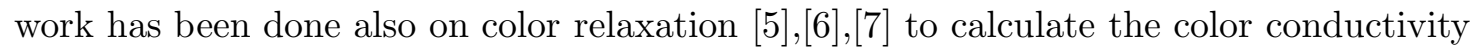
and the color diffusion coefficients.

We want to extend that work and calculate the static color conductivity $\sigma$ including a chemical potential for quarks. The chemical potential has been estimated to become zero only when nuclear transparency sets in and reach values up to $1-2$ times the temperature in the region where stopping occurs [8].

The (infrared) divergence occuring in the calculation of color conductivity, which can be related to long range (color) magnetic interactions, will be treated in our approach self-consistently by including a finite width $\nu$ for quarks and gluons which acts as a self-consistent damping term. In previous works [5], [6], [7] this divergence was regularized using a (non-perturbative) magnetic screening mass $m_{g}$ as an additional cutoff parameter. We will show explicitely, that a finite width will regularize the divergence self-consistently. 
The paper will be organized as follows: In section 2 we will get the self-consistent equations for the width $\nu$ of both gluons and quarks to first order perturbation theory in the hard thermal loop approximation using the real time formalism of thermal field dynamics (TDF) including a chemical potential $\mu$ for quarks. In section 3 we will discuss the self-consistent (numerical) solutions of $\nu$ and $\sigma$ as functions of temperature and chemical potential. We will summarize our results in section $甘$.

\section{Damping rates of fast moving particles in a QGP}

If one considers a fast moving particle with momentum scale $p \gg g T$ going through a weakly coupled QGP $(g \ll 1)$, the relativistic dispersion relation for the particle, the transverse gluon for instance, can be written according to the poles of its propagator in the following form

$$
E(k)=\omega(k)-i \nu_{T},
$$

where $\nu_{T}$ is the damping rate of the transverse gluon.

It is sufficient to consider only the fast moving particles since for the static color conductivity the momentum exchange is small $(\mathrm{O}(g T))$. This results in a damping rate for the slow moving particles of order $g^{2} T$ in the weak coupling limit, which is small compared to the one for the fast moving particles of order $g^{2} T / \ln (1 / g)$ [9]. This corresponds to the scenario of a realistic hot QGP, where the momentum of a particle should be of order $T$, that is hard $(\langle p\rangle=3 T)$, but where the momentum exchange can be rather small. In addition, the color diffusion coefficient $d_{c}=p \cdot u \nu_{T}$ is proportional to the momentum of the particle ( $u_{\mu}$ being the four velocity in the ensemble rest frame), so that color diffusion is suppressed for slow particles.

In covariant gauge the gluon propagator is

$$
G_{\mu \nu}(k)=A_{\mu \nu}(k) \Delta_{T}(k)+B_{\mu \nu}(k) \Delta_{L}(k)+D_{\mu \nu}(k) \Delta_{\xi}(k) .
$$

$\Delta_{T}$ and $\Delta_{L}$ are the transverse and longitudinal gluon propagators respectively, given by

$$
\Delta_{T, L}=\frac{1}{k^{2}-\Pi_{T, L}},
$$

$\xi$ is the gauge parameter with associated propagator

$$
\Delta_{\xi}=\xi \frac{1}{k^{2}}
$$

and $A_{\mu \nu}, B_{\mu \nu}, D_{\mu \nu}$ are functions of the loop momentum $k, k=\left(k_{0}, K\right)$,

$$
\begin{aligned}
A_{\mu \nu}(k) & =\delta_{\mu i}\left(\delta_{i j}-\frac{k_{i} k_{j}}{K^{2}}\right) \delta_{j \nu}, \\
B_{\mu \nu}(k) & =\left(\delta_{\mu 0}-\frac{k_{\mu} k_{0}}{k^{2}}\right) \frac{k^{2}}{K^{2}}\left(\delta_{\nu 0}-\frac{k_{\nu} k_{0}}{k^{2}}\right), \\
D_{\mu \nu}(k) & =\frac{k_{\mu} k_{\nu}}{k^{2}} .
\end{aligned}
$$

$\Pi$ is the polarization tensor, and the dispersion relation for the transverse gluon can be cast into the form [5]

$$
E^{2}-P^{2}=\frac{1}{2}\left(\Pi_{i}^{i}-\frac{p^{i} p^{j} \Pi_{i j}}{P^{2}}\right) \equiv F
$$


From Eqs.(11) and (6) one obtains the damping rate for a transverse gluon on the mass shell [5]:

$$
\nu_{T}=-\frac{\operatorname{Im} F}{2|P|} .
$$

The crucial step in evaluating Eq.(7) will be to calculate the imaginary part of the polarization tensor. The one-loop graphs for $\Pi$ in $S U(N)$ - QCD are given in Fig.1.

Since the imaginary part of the tadpole diagram vanishes, it does not contribute to the damping rate. For a fast moving particle with hard external momenta it is sufficient to use the effective propagator for one of the internal lines and bare propagators and vertices otherwise according to the resummation scheme of Braaten and Pisarski [9], [10]. Because of the Bose enhancement factor $1+n_{B}$ for the gluon loop, the diagram of Fig.1(a) will give the leading order contribution to the imaginary part of the polarization tensor, whereas the quark and ghost loop can be neglected due to the Pauli blocking factor $1-n_{f}$ and will contribute only to higher order in the coupling constant. Therefore up to leading order we only have to calculate the three-gluon one-loop resummation graph of Fig.1.(a).

In the static limit the contributions from the hard thermal loops for hot QCD are [8], [9], 10]

$$
\Pi_{L}\left(k_{0} \rightarrow 0, K\right)=\Pi_{00}\left(k_{0} \rightarrow 0, K\right)=m_{e l}^{2}=g^{2} T^{2}\left(1+\frac{N_{f}}{6}+\frac{1}{2 \pi^{2}} \sum_{f} \frac{\mu_{f}^{2}}{T^{2}}\right)
$$

for the longitudinal part and

$$
\Pi_{T}\left(k_{0} \rightarrow 0, K\right)=0
$$

for the transverse part of the gluon polarization tensor. Substituting Eqs.(8),(9) into Eq.(3), one gets

$$
\Delta_{L}=\frac{1}{k^{2}-m_{e l}^{2}}, \quad \Delta_{T}=\frac{1}{k^{2}} .
$$

The resummation method for perturbation theory at finite temperature as proposed by Braaten and Pisarski, which resums HTL contributions, works well if the momentum scale is $g T$. But in non-abelian gauge theories there is another pernicious barrier for perturbation theory besides the weak coupling limit: Static magnetic fields are not screened at the HTL level and this may lead to a breakdown of perturbation theory at high loop order. The corresponding infrared singularities are usually expected to be cured by the dynamical generation of a magnetic screening mass $m_{g}\left(g^{2} T\right)$ [5], [6], [7], but the nature of the latter is still unclear.

It is known that damping of collective modes in a QGP occurs. This dynamical screening, as opposed to static (Debye) screening, makes it reasonable to consider a damping rate for gluons, that is to assign them a finite width when studying the collective behaviour of a QGP. Assuming that the damping rate of the transverse gluon is $\nu_{T}$, we can write the transverse gluon propagator as [1], [12]

$$
\Delta_{T}^{\prime}=\frac{1}{\left(k_{0}-i \nu_{T}\right)^{2}-K^{2}}
$$

so that the total resummed gluon propagator becomes

$$
G_{\mu \nu}^{\prime}(k)=A_{\mu \nu}(k) \Delta_{T}^{\prime}(k)+B_{\mu \nu}(k) \Delta_{L}(k)+D_{\mu \nu}(k) \Delta_{\xi}(k) .
$$


The bare propagator and vertex are given by

$$
\begin{aligned}
\Delta_{\mu \nu}(k) & =-\frac{1}{k^{2}}\left(g_{\mu \nu}-(1-\xi) \frac{k_{\mu} k_{\nu}}{k^{2}}\right), \\
\Gamma_{\mu \nu \rho}(p, q, k) & =(p-q)_{\rho} g_{\mu \nu}+(q-k)_{\mu} g_{\nu \rho}+(k-p)_{\nu} g_{\mu \rho} .
\end{aligned}
$$

By making use of Eqs.(11)-(14), one can evaluate the diagram of Fig.11(a) and obtain for $F$ from Eq.(6) after a rather lengthy calculation

$$
F=g^{2} N_{c} \int \frac{d^{D} q}{(2 \pi)^{D}} \frac{4|P|^{2}}{(p+q)^{2}} \cdot\left(\Delta_{T}^{\prime}(q)-\Delta_{L}(q)-(1-\xi) \frac{(\hat{P} \cdot \hat{Q})^{2}}{q^{2}}\right),
$$

where the hats on the three-vectors $P$ and $Q$ denote unit vectors.

In the following we will apply the formalism of thermal field dynamics (TFD), since the imaginary part of the " $1-2$ " component of the Greens function $\Sigma_{12}$ is easier evaluated in TFD than in the imaginary time formalisme. By using the well known relation between the imaginary parts of retarded Greens functions and "1 - 2" components of Greens functions in TFD [13, 14,

$$
\operatorname{Im} \bar{\Sigma}=-\frac{e^{\beta\left|p_{0}\right|}-1}{2 e^{\beta\left|p_{0}\right| / 2}} \operatorname{Im} \Sigma_{12}
$$

one immediately obtains

$$
\begin{aligned}
\operatorname{Im} F= & -g^{2} N_{c} \frac{e^{\beta\left|p_{0}\right|}-1}{2 e^{\beta\left|p_{0}\right| / 2}} \int \frac{d^{D} q}{(2 \pi)^{D}} 4|P|^{2} \Delta_{12}(p+q) \\
& \cdot\left(\Delta_{T}^{12}(q)-\Delta_{L}^{12}(q)-(1-\xi)(\hat{P} \cdot \hat{Q})^{2} \Delta_{12}(q)\right) .
\end{aligned}
$$

The thermal propagators of $1-2$ type have the form 14

$$
\begin{aligned}
\Delta_{L}^{12}(q) & =2 \pi i \delta\left(q^{2}-m_{e l}^{2}\right) \cdot n_{B}(q) e^{-\beta\left|q_{0}\right| / 2} \\
\Delta_{T}^{12}(q) & =2 \operatorname{Im} \Delta_{T} \\
& =\left[\frac{1}{\left(q_{0}-i \nu_{T}\right)^{2}-Q^{2}-m_{g}^{2}}-\frac{1}{\left(q_{0}+i \nu_{T}\right)^{2}-Q^{2}-m_{g}^{2}}\right] \cdot n_{B}(q) e^{-\beta\left|q_{0}\right| / 2} \\
& \stackrel{\nu_{T}}{ }=0 \\
& =2 \pi i \delta\left(q^{2}-m_{g}^{2}\right) \cdot n_{B}(q) e^{-\beta\left|q_{0}\right| / 2} \\
\Delta_{12}(q) & =2 \pi i \delta\left(q^{2}\right) \cdot n_{B}(q) e^{\beta\left|q_{0}\right| / 2}
\end{aligned}
$$

We included the magnetic mass $m_{g}$ in the propagators, since both self-consistent damping and magnetic screening are non-perturbative effects of order $g^{2} T$ so that one cannot neglect a priori one effect compared to the other. In addition, including $m_{g}$ will enable us to compare our self-consistent results with the previous results, where the infrared divergence was regularized by a magnetic mass $m_{g}$ alone.

Eq.(18) contains the Bose-Einstein distribution function $n_{B}$, which for soft momentum, $q \ll p \sim T$, reduces to

$$
n_{B}(q)=\frac{1}{e^{\beta\left|q_{0}\right|}-1} \simeq \frac{T}{\left|q_{0}\right|}, \quad n_{B}(p+q) \simeq n_{B}(p) .
$$


Substituting Eqs.(18)-(21) into Eq.(17), one can evaluate the integration by dimensional regularization at finite temperature [15]. The final result is

$$
\operatorname{Im} F=-\frac{g^{2} N_{c} T|P|}{4 \pi}\left(\ln \frac{m_{e l}^{2}}{m_{g}^{2}+\nu_{T}^{2}}+1-\frac{2}{\pi} \arctan \frac{m_{g}}{\nu_{T}}+\mathcal{O}\left(g^{2}\right)\right) .
$$

From the definition Eq.(7]) one obtains

$$
\nu_{T}=-\frac{\operatorname{Im} F}{2|P|}=\frac{g^{2} N_{c} T}{8 \pi}\left(\ln \frac{m_{e l}^{2}}{m_{g}^{2}+\nu_{T}^{2}}+1-\frac{2}{\pi} \arctan \frac{m_{g}}{\nu_{T}}\right) .
$$

We can rewrite Eq.(23) in terms of a new energy scale defined by $m_{g}$ with dimensionless variables $\bar{\nu}_{T}=\nu_{T} / m_{g}, \bar{T}=T / m_{g}, \bar{\mu}_{T}=\mu_{T} / m_{g}$ and

$$
\bar{m}_{e l}=\frac{m_{e l}}{m_{g}}=g \bar{T}\left(1+\frac{N_{f}}{6}+\frac{1}{2 \pi} \sum_{f} \frac{\bar{\mu}_{f}^{2}}{\bar{T}^{2}}\right)^{1 / 2}
$$

as

$$
\bar{\nu}_{T}=\frac{g^{2} N_{c} \bar{T}}{8 \pi}\left(2 \ln \bar{m}_{e l}-\ln \left(1+\bar{\nu}_{T}^{2}\right)+1-\frac{2}{\pi} \arctan \frac{1}{\bar{\nu}_{T}}\right) .
$$

This is a self-consistent equation for the rescaled damping rate $\bar{\nu}_{T}$ and it is gauge independent to leading order in the coupling constant.

If one neglects the finite width effects for the internal particles $\left(\bar{\nu}_{T} \ll 1\right)$ Eq.(25) yields

$$
\bar{\nu}_{T}=\frac{g^{2} N_{c} \bar{T}}{8 \pi} \ln \bar{m}_{e l}^{2}
$$

which recovers the previous results from Refs. [5], [7], [16].

If on the other hand selfconsistent damping dominates magnetic screening $\left(\bar{\nu}_{T} \gg 1\right)$ Eq.(25) reduces to

$$
\bar{\nu}_{T}=\frac{g^{2} N_{c} \bar{T}}{8 \pi}\left(\ln \frac{\bar{m}_{e l}^{2}}{\bar{\nu}_{T}^{2}}+1\right)
$$

which can be rewritten as

$$
\nu_{T}=\frac{g^{2} N_{c} T}{8 \pi}\left(\ln \frac{m_{e l}^{2}}{\nu_{T}^{2}}+1\right)
$$

where $m_{g}$ drops out completely. This again is a selfconsistent equation for $\nu_{T}$ and the infrared divergences encountered in previous calculations are removed self-consistently.

The selfconsistent Eqs. (25) and (27) will prove to have very similar solutions for $\bar{T} \geq 1$, but differ from the solutions for Eq. (26).

One can calculate the damping rate for a fast quark going through a QGP at finite temperature and chemical potential in a way similar to the preceeding calculation for gluons by evaluating

$$
\nu_{q}=-\left.\frac{1}{4|p|} \operatorname{Im} \operatorname{Tr}(\not p \Sigma)\right|_{p_{0}=E} .
$$

Let us consider first the quark self-energy shown in Fig.2, where we have included screening and damping rate effects by means of the effective gluon propagator [9]. For 
hard quarks $\left(\left\langle p_{q}\right\rangle \sim T\right)$ it is sufficient to use the bare quark propagator and bare vertices in the hard thermal loop approximation.

We will calculate the imaginary part of the self-energy at finite temperature and chemical potential using TFD. For the hard particles one can show that the main contribution to the interaction rate comes from the soft momentum transfer range, i.e. $q \sim g T$ in the weak coupling limit.

Applying the usual Feynman rules of QCD to Fig.2, one obtains

$$
\operatorname{Tr}(\not p \Sigma) \equiv H=-g^{2} C_{f} \int \frac{d^{D} q}{(2 \pi)^{D}} \frac{\operatorname{Tr} \not \gamma^{\mu}(\not p+\not 1) \gamma^{\nu}}{(p+q)^{2}} G_{\mu \nu}
$$

with $C_{f}=\frac{N^{2}-1}{2 N}$ the Casimir for $S U(N)$. By using Eqs.(2)-(5), Eq.(30) can be cast into the form

$$
H=-g^{2} C_{f} \int \frac{d^{D} q}{(2 \pi)^{D}} \frac{2|P|^{2}}{(p+q)^{2}}\left(\Delta_{T}^{\prime}(q)-\Delta_{L}(q)-(1-\xi) \frac{(\hat{P} \cdot \hat{Q})^{2}}{q^{2}}\right)
$$

Then using the hot propagators in TFD for gluons as given by Eq.(18) and for quarks as follows

$$
\begin{aligned}
& i \Delta_{11}(p)=-i \Delta_{22}(p)=\not\left[\frac{1}{p^{2}-i \epsilon}-2 \pi\left(\theta\left(p_{0}\right) e^{x_{p} / 2} n_{f}\left(x_{p}\right)+\right.\right.\left.\left.\theta\left(-p_{0}\right) e^{-x_{p} / 2} n_{f}\left(-x_{p}\right)\right) \delta\left(p^{2}\right)\right], \\
& i \Delta_{12}(p)=-i \Delta_{21}(p)=-2 \pi p e^{-\beta \mu}\left[\theta\left(p_{0}\right) e^{x_{p} / 2} n_{f}\left(x_{p}\right)+\right. \\
&\left.\theta\left(-p_{0}\right) e^{-x_{p} / 2} n_{f}\left(-x_{p}\right)\right] \delta\left(p^{2}\right),
\end{aligned}
$$

where $n_{f}\left(x_{p}\right)=\left[e^{x_{p}}+1\right]^{-1}$ with $x_{p}=\beta\left(p_{0}+\mu\right)$ denotes the Fermi-Dirac distribution function, we obtain

$$
\begin{aligned}
\operatorname{Im} H= & -g^{2} C_{f} \int \frac{d^{D} q}{(2 \pi)^{D}} e^{-\beta \mu / 2} 2|P|^{2} \sin 2 \phi_{p_{0}^{\prime}+\mu}(2 \pi)^{2} \\
& \left(\Delta_{T}^{12}(q)-\Delta_{L}^{12}(q)-(1-\xi) \cos ^{2} \theta \Delta_{12}(q)\right) . \\
= & 4 g^{2} C_{f} T \int \frac{d^{D-1} q}{(2 \pi)^{2}}|P|^{2} \delta\left((p+q)^{2}\right) \\
& \cdot\left(\frac{1}{Q^{2}+\nu^{2}}-\frac{1}{Q^{2}+m^{2}}-(1-\xi) \frac{\cos ^{2} \theta}{Q^{2}}\right) .
\end{aligned}
$$

Here we used the following relation between the retarded self-energy $\bar{H}$ and the 1-2 component of the self-energy $H_{12}$ in TFD

$$
\operatorname{Im} \bar{H}=\frac{e^{\beta \mu / 2}}{\sin 2 \phi_{p_{0}+\mu}} \operatorname{Im} H_{12}
$$

where

$$
\begin{aligned}
\cos \phi_{p_{0}+\mu} & =\frac{\theta\left(p_{0}\right) e^{x_{p} / 4}+\theta\left(-p_{0}\right) e^{-x_{p} / 4}}{\sqrt{e^{x_{p} / 2}+e^{-x_{p} / 2}}} \text { and } \\
\sin \phi_{p_{0}+\mu} & =\frac{\theta\left(p_{0}\right) e^{-x_{p} / 4}+\theta\left(-p_{0}\right) e^{x_{p} / 4}}{\sqrt{e^{x_{p} / 2}+e^{-x_{p} / 2}}} .
\end{aligned}
$$


Evaluating the integration in Eq.(34) by dimensional regularization at finite temperature [15] we get as the final result for the width of the quarks

$$
\nu_{q}=\frac{g^{2} C_{f} T}{8 \pi}\left(\ln \frac{m_{e l}^{2}}{m_{g}^{2}+\nu_{T}^{2}}+1-\frac{2}{\pi} \arctan \frac{m_{g}}{\nu_{T}}\right) .
$$

Rewritting Eq.(38) as before in terms of dimensionless variables $\bar{\nu}_{q}=\nu_{q} / m_{g}, \bar{\nu}_{T}, \bar{T}$, $\bar{m}_{e l}$ leads to

$$
\bar{\nu}_{q}=\frac{g^{2} C_{f} \bar{T}}{8 \pi}\left(2 \ln \bar{m}_{e l}-\ln \left(1+\bar{\nu}_{T}^{2}\right)+1-\frac{2}{\pi} \arctan \frac{1}{\bar{\nu}_{T}}\right)
$$

which gives $\bar{\nu}_{q}$ as a function of $\bar{\nu}_{T}$ and $\bar{T}$, where $\bar{\nu}_{T}$ is determined self-consistently through Eq.(25).

Setting the finite width of the internal particle to zero, one recovers the result given in $[5]$.

One thus finds that the damping rates for the quark and gluon have the same behaviour with temperature and chemical potential to leading order in the coupling constant and differ only by a constant factor $C_{f} / N_{c}$, which counts the number of processes available for color relaxation for quarks as compared to gluons.

\section{Numerical Analysis of Color Conductivity for a QGP}

In a QGP the interaction rates or frequencies among particles should be sensitive to variations of temperature and baryon density. Naively one might expect the interaction rates to increase with increasing temperature $T$ or chemical potential $\mu$, but one can see from the self-consistent Eqs.(23),(38) that the interaction rates depend strongly only on temperature. The reason for this is that the chemical potential $\mu$ reflects only the baryon density in a QGP and does not describe the color charge density.

We solved numerically the self-consistent Eqs.(23),(38) for the gluon and quark damping rates as functions of temperature for different values of the chemical potential in the weak coupling limit $\left(\alpha_{s}=0.1\right)$ for two quark flavors.

Changing back from quantities $\bar{\nu}, \bar{T}$, where the energy scale is set by $m_{g}$ to ordinary temperature $T$ and damping rate $\nu$ we have to make an ansatz for the magnetic screening mass $m_{g}$, which we know to be of order $g^{2} T$ :

$$
m_{g} \equiv c \alpha_{s} T \quad \text { with } \alpha_{s}=\frac{g^{2}}{4 \pi} .
$$

The dependence of Eqs. (23), (38) on $c$ is given by a linear decrease of the asymptotic slope with increasing $m_{g}$ for fixed $T, \Delta \nu_{T} /\left.\Delta c\right|_{T=\text { const }}=-\pi \alpha_{s} T=$ const. For Eq. (26) on the other hand the change of the slope of $\nu_{T}$ as a function of $c$ is given by $\Delta \nu_{T} / \Delta c=-3 / c$, so that for large $c$ the asymptotic slope does practically not change.

In the following we will adopt a value of $c=3$ as estimated from lattice gauge theories 17] and classical considerations [18.

For the considered values of $T$ around $200 \mathrm{MeV}$ in Fig. 3 we find an only slightly flater curve for Eq.(23), where the IR-singularities were regularized through a combination of selfconsistent damping and magnetic screening with $m_{g}=3 \alpha_{s} T$ compared to Eq.(28), 
where selfconsistent damping alone was used. The latter case (limit $\nu_{T} \gg 1$ ) corresponds to $c=0$, that is vanishing $m_{g}$ in Eq.(23).

For Eq.(26) on the other hand, where the IR-singularities were regularized through a magnetic screening mass alone we find a steeper asymptotic slope.

The results as given in Fig. 3 show that, as expected, the damping rate is not sensitive to the changes of the chemical potential, and only slightly enhanced with increasing $\mu$. Otherwise it is approximately proportional to temperature.

The influence of chemical potential is highest at very low temperature, corresponding to a cold (and dense) QGP, like for instance the core of a neutron star. For hot and dilute QGP as expected from ultrarelativistic heavy ion collisions the influence of chemical potential on the damping rate becomes negligible.

From the transport theory of the QGP one can derive the static color conductivity as given in [6],[7]:

$$
\sigma_{i}=\frac{\omega_{p l}^{2}}{\nu}, \quad \omega_{p l}^{2}=m_{e l}^{2} / 3
$$

The color conductivity describes the ability of color transport in a QGP. It is defined as the ratio between the plasma frequency squared and the interaction rate $\Gamma$ of the particles in a QGP, where $\Gamma$ is the inverse color relaxation time and therefore given by the damping rate $\nu$. Since the interaction rate increases only linearly with temperature and logarithmically with chemical potential, but the plasma frequency squared increases quadratically with $T$ as well as $\mu$, color conductivity is enhanced with increasing $T$ and $\mu$.

As a numerical results for $\sigma$ one obtains the curves given in Fig. 4 by using the selfconsistent solutions for the damping rates. One finds that the quark chemical potential has some influence on the color conductivity, especially at low temperatures where our assumption of a finite chemical potential does apply.

\section{Discussion and Conclusions}

In summary, by taking into account the quark chemical potential and the transverse gluon damping effects, we obtain the self-consistent equation for the damping rate of gluons and quarks in real time finite temperature QCD language, where the infrared divergence is removed self-consistently and the magnetic screening mass leads to a redefinition of the energy scale.

In addition we have solved the equation for the damping rate numerically and calculated the color conductivity for a QGP for different chemical potentials with an ansatz for the magnetic screening mass $m_{g}$. The results show that the chemical potential enhances the color conductivity somewhat but has practically no effect on the damping rate for gluons and quarks.

Without considering a chemical potential or self-consistent damping we can recover the results previously derived by other groups.

We conclude that the quark chemical potential has influence on color transport only at low temperatures and high densities. Except for low temperatures color conductivity rises linearly with $T$, where the slope of the curves depends on the mechanisme to regularize the IR divergences. Because of this $T$ dependence dissipation can not be neglected in 
hydrodynamic descriptions of the expansion phase of a hot QGP in ultrarelativistic heavy ion collisions.

This work is supported partly by the National Natural Science Funds of China. S.Ochs wants to thank the Deutsche Forschungsgemeinschaft (DFG), the Bundesministerium für Bildung und Forschung (BMBF), and the Gesellschaft für Schwerionenforschung (GSI) for their support. The authors are indebted to P. Henning for stimulating discussions and wish to thank Prof. Liu Lianshou and Prof. U.Heinz for their help. 


\section{$5 \quad$ Figure captions}

Figure 1: The resummed one-loop gluon self-energy graphs: (a) tadpole, (b) gluon loop, (c) fermion loop, (d) ghost loop.

Figure 2: The resummed one-loop quark self-energy graph.

Figure 3: Damping rate $\nu_{T}$ for gluons versus temperature $T$ for different quark chemical potentials, with $\alpha_{s}=0.1$. Dashed lines: without selfconsistent damping for three different chemical potentials $\mu=0,0.5,1.0 \mathrm{GeV}$ from bottom to top. Dotted lines: selfconsistent damping without magnetic screening, chemical potential $\mu$ as before. Solid lines: selfconsistent damping and magnetic screening, $\mu$ as before.

Figure 4: Color conductivity $\sigma$ versus temperature $T$ for different quark chemical potentials, with $\alpha_{s}=0.1$. Coding of lines as in Fig. 3. 


\section{References}

[1] B. Müller, "Physics of Quark-Gluon-Plasma" in: Proceedings of the Workshop on Pre-equilibrium Parton Dynamics, ( Ed. Xingnian Wang ), Berkeley, California, 1994; J.-P. Blaizot, Nucl. Phys. A566, (1994):333c.

[2] U. Heinz, K. Kajantie and T. Toimela, Ann. Phys. 176, (1987):218; U. Heinz Ann. Phys. 168, (1986):148.

[3] P. Danielewicz and M. Gyulassy, Phys. Rev. D31, (1985):53, Phys. Rev. D45, (1992):R17.

[4] G.Baym, et al. Phys. Rev. Lett. 64, (1990):1867; M.H. Thoma and M. Gyulassy, Nucl. Phys. B351, (1991):491.

[5] C.P. Burgess and A.L. Marini, Phys. Rev. D45, (1992):17.

[6] A.V. Selikov and M. Gyulassy, Phys. Lett. B316, (1993):373.

[7] M.H. Thoma, Phys. Rev. D49, (1994):451, Phys. Lett. B216, (1991):144.

[8] H. Vijia and M.H. Thoma, Phys. Lett. B342, (1995):212.

[9] E. Braaten and R.D. Pisarski, Phys. Rev. D42, (1990):2156, Phys. Rev. Lett. 64, (1990):1338.

[10] R.D. Pisarski, Nucl. Phys. A525, (1991):175c, Phys. Rev. Lett. 63, (1989):1129.

[11] N.P. Landsman and Ch.G. Van Weert, Phys. Rep. 145, (1987):141.

[12] P. Henning, Phys. Rep. 253, (1995):325.

[13] Y. Fujimoto, M. Morikawa and M. Sasaki, Phys. Rev. D33, (1986):590.

[14] R. Kobes, Phys. Rev. D42, (1990):562, Phys. Rev. D43, (1991):1269.

[15] Y. Fujimoto and Hou Defu, Phys. Lett. B335, (1994):87.

[16] A. Rebhan, Phys. Rev. D48, (1993):R3967; E. Braaten and A. Nieto, Phys. Rev. Lett. 73, (1994):2402.

[17] T.A. deGrand and D. Toussaint, Phys. Rev. D 25 (1982) 526.

[18] T.S. Biró and B. Müller, Nucl. Phys. A561, (1993) 477. 

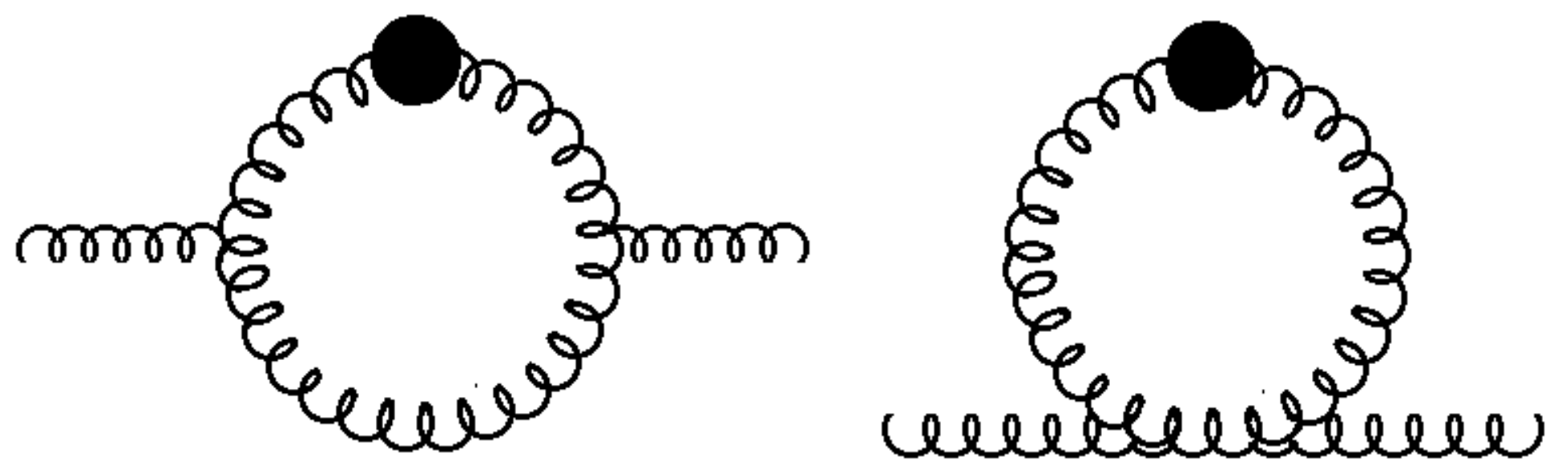

Fig. 1 (a)

Fig. 1 (b)
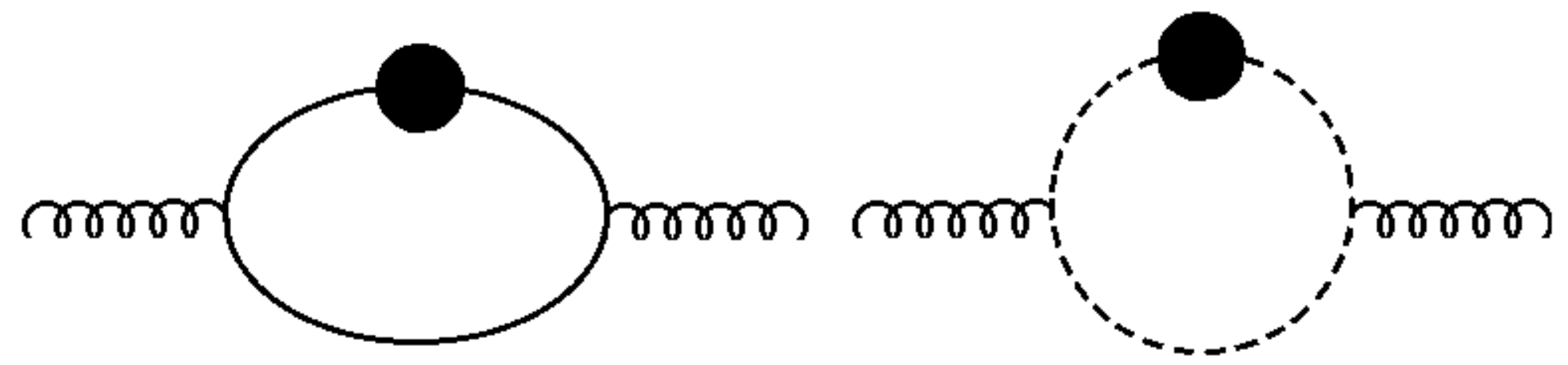

Fig. 1(c)

Fig. 1 (d) 


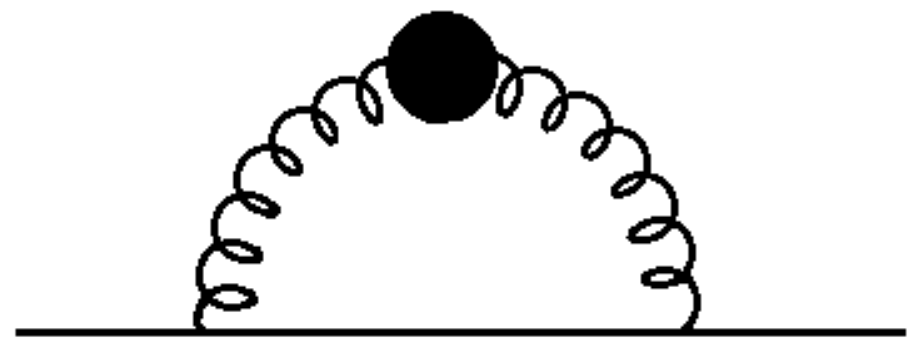

Fig. 2 


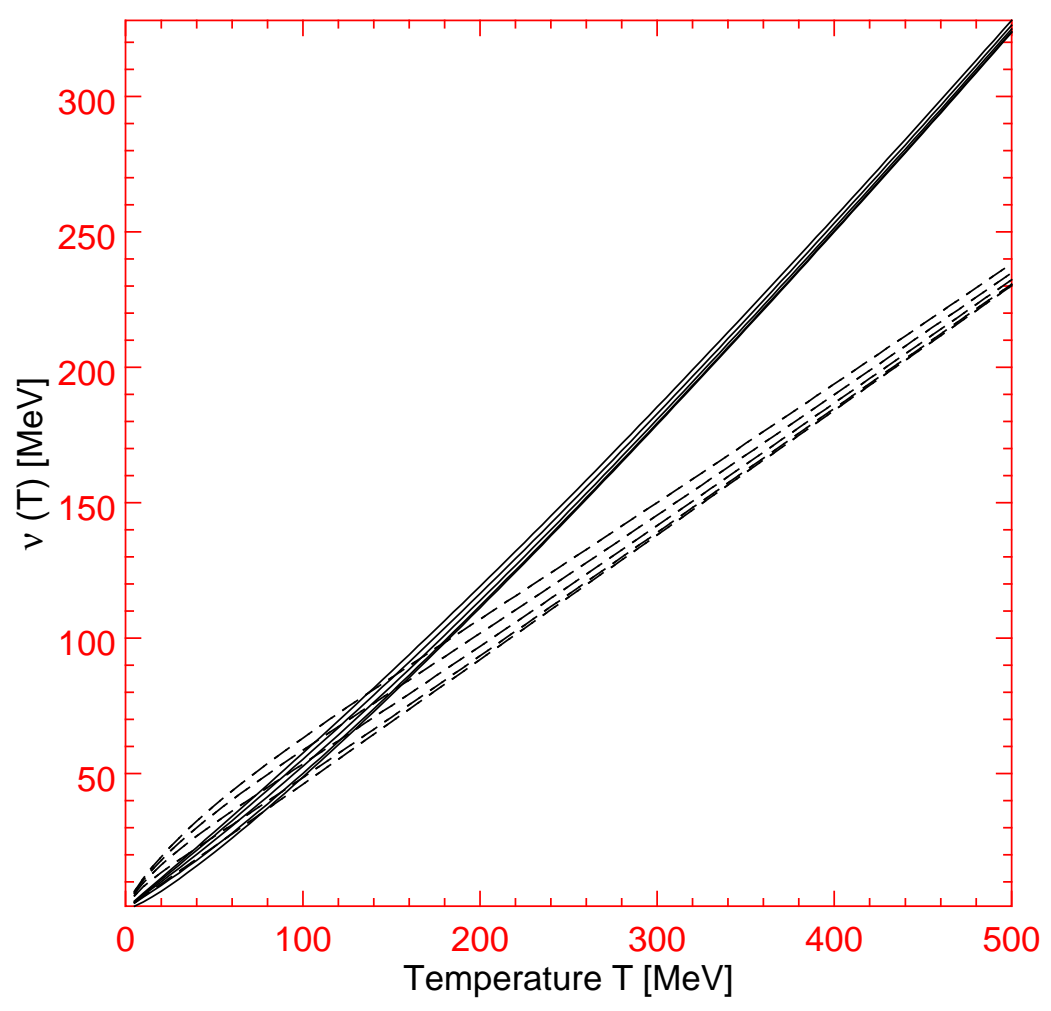

Fig. 3 


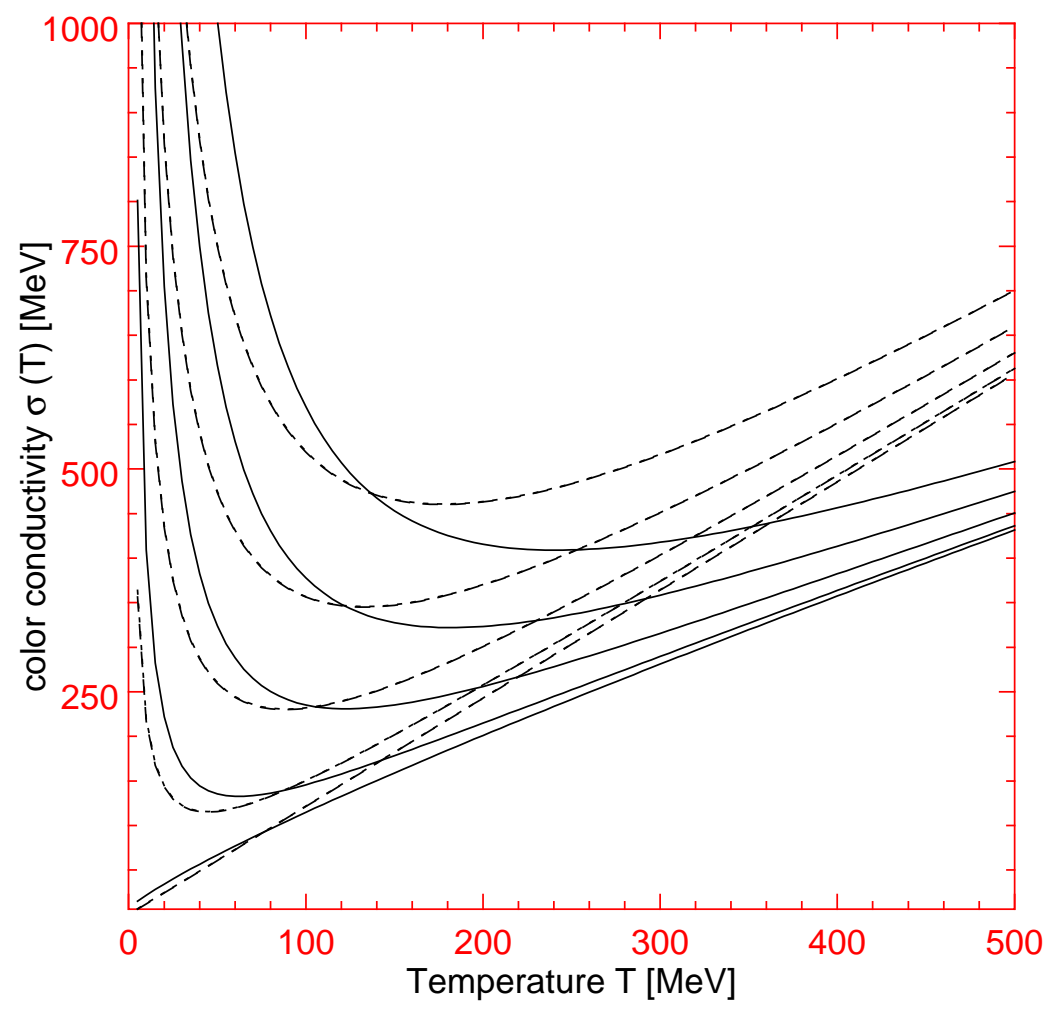

Fig. 4 